\title{
SMALL FOOTPRINT KNIFE GATE MICROVALVES FOR LARGE FLOW CONTROL
}

\author{
Stefan Braun, Sjoerd Haasl, Samir Sadoon, Anthony S. Ridgeway, Wouter van der Wijngaart, Göran Stemme \\ Microsystem Technology, Department of Signals, Sensors and Systems, \\ Royal Institute of Technology, Stockholm, Sweden \\ email: Stefan.Braun@s3.kth.se
}

\begin{abstract}
This paper introduces the first area-optimized micromachined knife gate microvalve. In comparison to recent microvalves the pressure-flow performance is increased using out-of-plane actuators and an out-ofplane orifice. Three different actuator-gate designs and their fabrication are described. The valve features integrated thermal silicon/aluminum bimorph actuators where the aluminum layer forms the resistive heater as well as the bimorph material. The characterization of the actuators and of the pressure-flow performance is presented. The valve allows a flow change of $\Delta Q=3.4 \mathrm{l} / \mathrm{min}$ at $100 \mathrm{kPa}$ on an active chip area of only $2.3 \times 3.7 \mathrm{~mm}^{2}$.
\end{abstract}

Keywords: Microvalves, Micropneumatics, Microfluidics

\section{INTRODUCTION}

The ability of controlling large gas flows with microvalves is of big interest due to technical and costrelated advantages. The technical advantages include the rapid response time, the low power consumption and the small device size. Manufacturing cost is an issue of major importance. MEMS offers batch fabrication and therewith an increased cost-efficiency. The latter is mainly defined by the number of devices per wafer and the simplicity of processing. The greatest advantage of microvalves, however, lies in the integration of the actuation mechanism with the other microvalve components, which enhances miniaturization and packaging [1].

So far microvalves are not used as standard components because of some major difficulties. Many of today's gas microvalves are of the membrane type and their actuator needs to counteract the static pneumatic force of the gas, so the maximum pressure allowed is defined by the strength of the actuator. To allow a large gas flow a valve stroke of several tens of micrometers is needed, which requires large actuator size and increased footprint area. There have becn attempts to minimize the required actuator force for membrane-type microvalves involving pressure balancing schemes [2], [3] or nozzle/seat optimization [4], [5]. Even if the actuator size could be reduced drastically, the minimization of the device area is still limited by the required minimum in-plane flow orifice.
Walters et al, [6] introduced the gate microvalve approach. Gate valves feature sliding flow gates which move perpendicular to the flow direction, minimizing the demands on the actuator. The improved performance of gate valves comes with a compromise. MEMS actuators do not allow friction between sliding structures. The required spacing between the flow orifice and the regulating gate results in a leak flow in the closed state of the valve. However, the intended application of the valve described below tolerates leak flow and benefits from the advantages of decreased actuator demands and increased flow performance. This paper introduces knife gate microvalves that feature out-of-plane orifices and pneumatic interfaces at the side of the silicon chip. The design is based on a principle first introduced by our group in [7].

\section{DESIGN AND APPLICATION}

We introduce knife gate microvalves to cireumvent the above stated problems. A gate moves out-of-plane and regulates the in-plane flow through the orifice (see Figure 1). This enables a smaller actuator and less footprint area. The substrate material is not structured and can be any flat material.

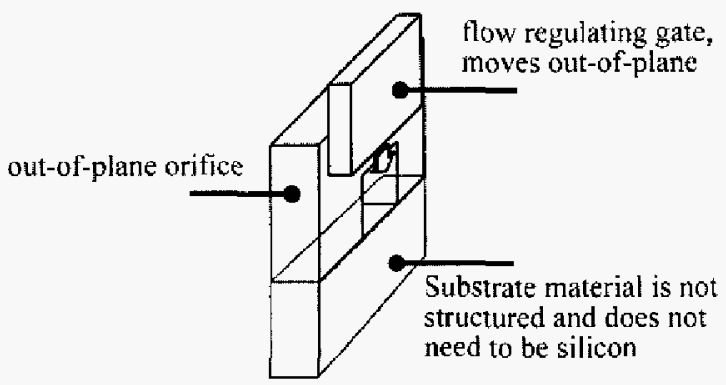

Figure 1: Principle of the knife gate microvalve

We propose three basic configurations for normally-open knife gate microvalves, hereafter referred to as front gate valve (FG), side gate valve (SG) and back gate valve (BG) (see Figure 2). These designs differ in the relative position between the bimorph actuator and the mean flow direction. 


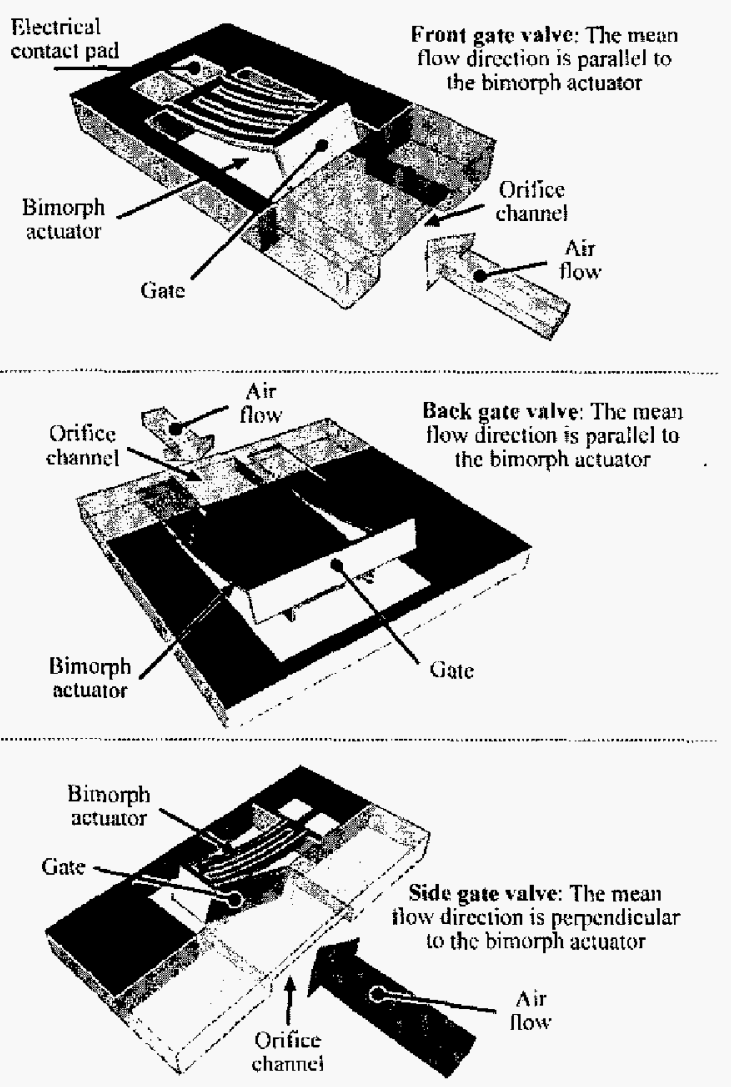

Figure 2: The illustrations show the three different valve configurations and their components. The channels are illustrated transparently for clarity (not to scale).

The channel cross-sectional area determines the pressure/flow characteristics and defines the pneumatic force on the closed gate. For the side gate valve the pressure force is orthogonal to the beam and induces torsional forces along the beam. The back gate and front gate valve offer a better mechanical stability since the flow is paraliel to the beam and no torsional forces along the beam are created.

Friction between sliding microstructures must be avoided. Therefore there has to be a gap between the gate and the orifice which results in a closed-state leakage. Microfabrication allows one to minimize the gap and therewith the closed-state leakage. For the side gate and back gate valve this leakage gap is only determined by the manufacturing process. For the front gate valve the gap is determined by the operation, not by the fabrication. The latter design requires the largest orifice-gate spacing, due to the outward rotation of the gate during upward movement, which results in a higher closed-state leakage.

The back gate valve design has the largest footprint area of the three designs but compensates by combining two major advantages: a leakage gap determined by fabrication rather than operation and the best mechanical stability, so it is the most promising design. Table 1 shows a comparison between the three designs.

Table 1: A comparison of the properties for the different valve configurations

\begin{tabular}{|c|c|c|c|}
\hline & Side gate & Front gate & Back gate \\
\hline $\begin{array}{l}\text { Minimal leak gap } \\
\text { defined by }\end{array}$ & Fabrication & Operation & Fabrication \\
\hline $\begin{array}{l}\text { Orifice chandel } \\
\text { footprint }\end{array}$ & Small & Small & Large \\
\hline $\begin{array}{l}\text { Longitudinal } \\
\text { torsion }\end{array}$ & Large & Small & Small \\
\hline Minimum area & Small & Medium & Large \\
\hline
\end{tabular}

The present knife gate microvalve is intended for application as the core of an IP-controller. IPcontrollers convert an electrical current, I, into a pneumatic pressure, P. Such controllers feature three pneumatic ports: a supply-, a vent- and a work port. Figure 3 illustrates how two front gate valves can be combined to form a pressure controller. By controlling the two valves the work pressure can be regulated between the supply pressure and the atmospheric pressure.
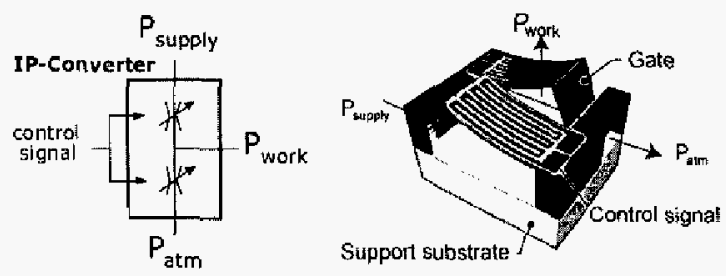

Figure 3: Combination of two cross-flow valves to form an $\mathrm{L} / \mathrm{P}$ converter.

\section{FABRICATION}

We fabricated prototypes of the three designs. Figure 4 shows the four major fabrication steps. In the first step the wafer is wet oxidized to create a $1 \mu \mathrm{m}$ thick oxide layer. Then the top side oxide is etched away and regrown to obtain a $100 \mathrm{~nm}$ layer to electrically insulate the silicon from the subsequently sputtered $3 \mu \mathrm{m}$ thick aluminum layer. The aluminum is patterned and wet etched to form the heaters. After this, the back side is patterned in two steps to define the orifice channels and bimorph beams. The first mask defines the bimorph beams and the orifice channels and is etched into the oxide. The second mask covers the orifice channels with thick resist. This is the situation at (a). The back side is then etched using DRIE in two steps, where the thick resist for the second mask is removed halfway, causing the orifice channels to be etched only during the second step. This is shown in (b) and (c). Finally, a front side DRIE frees the devices 
and a BHF etch removes all unnecessary oxide. In some cases, RIE lag hindered the complete etching of the narrow gap between the gate and the orifice channel. In those cases, an additional DRIE from the front was performed to "cut" free the gates. After this, the devices were diced. The final device, is shown in (d). Figure 5 shows a fabricated back gate microvalve.

(a)

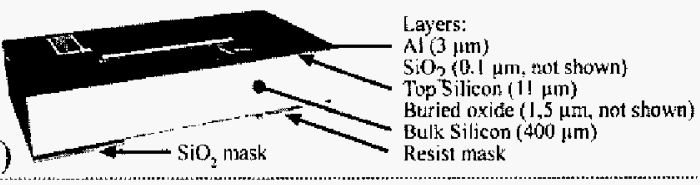

(b)

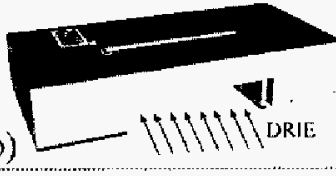

$\mathrm{SiO}$, mask for DRIE - step Orifice channel is covered with resis

(c)

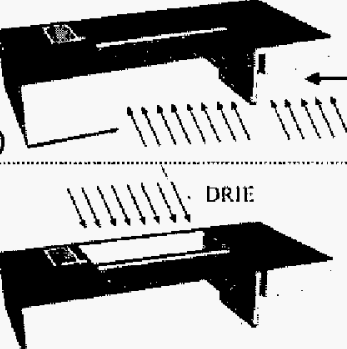

Resist removed halfway, orifice channel gets etched

(d)

Figure 4: Microfabrication process flow for the knife gate microvalve, illustrated for the front gate valve.

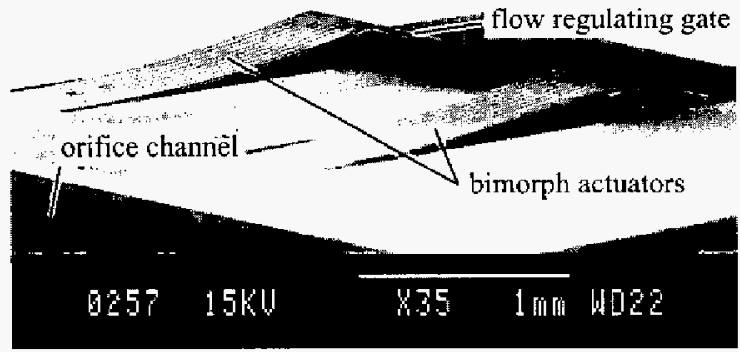

Figure 5: SEM Photograph of a backside gate valve with an opening of approximately $120 \mu \mathrm{m}$.

\section{MEASUREMENTS}

A back gate valve was connected pneumatically according to the scheme in Figure 6. First the chip was glued to a glass substrate, then a brass tube connector was glued to its side. This assembly method was evaluated by ramping up the pressure until the assembly ruptured, which occurred at 6 bars.

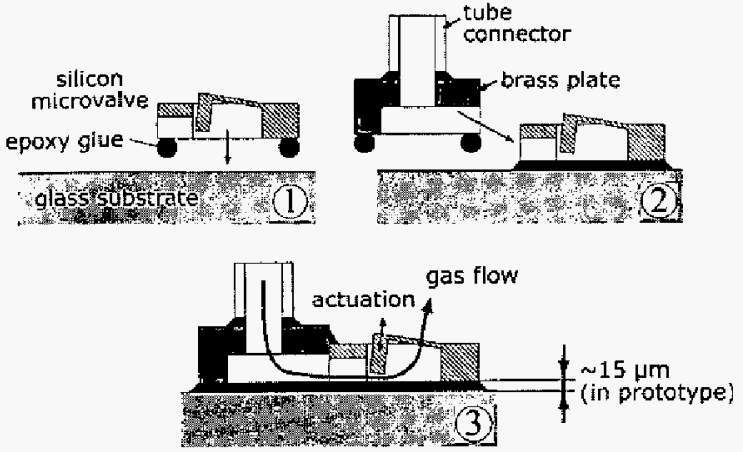

Figure 6: Cross-sectional view of the assembly of the pneumatic interconnection at the sides of the chip reducing the required silicon area.

The aluminum was deposited at a temperature of $120^{\circ} \mathrm{C}$, so one would expect the actuator to be fully actuated at this temperature. We tested the thermal bimorph actuator by heating with the integrated aluminum resistors, and by heating the complete component with a hotplate, respectively. Whereas external hotplate heating closes the valve completely at $120^{\circ} \mathrm{C}$, this is not the case for heating with the integrated resistors. This is due to a lack of thermal insulation of the actuator. Resistive heat is dissipated to the silicon bulk material, resulting in an inhomogeneous heat distribution along the bimorph: whereas the end of the cantilever reaches the maximum temperature aluminum can withstand $\left(660^{\circ} \mathrm{C}\right)$, the clamping side is not heated enough. By heating with a hotplate the whole device is at the same temperature, so the actuation works as expected. Measurement curves for both tests are compared in Figure 7.

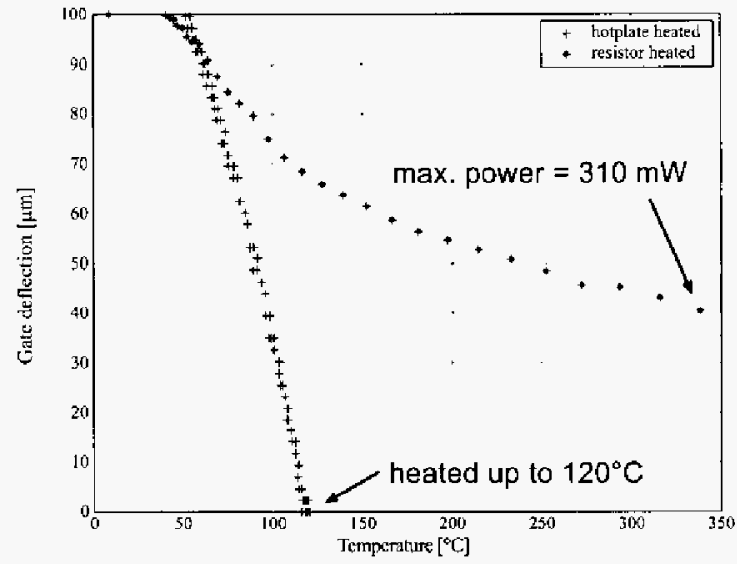

Figure 7: Measured deflection of a bimorph actuator using a hotplate respectively the integrated resistor to heat the device. The deviation shows the need for improved thermal isolation. 
To evaluate the pressure-flow characteristics, an external manipulator was used to actuate the gate. The measurements (see Figure 8) show the valve's potential for controlling large flows: a flow orifice size of $1000 \times 200 \mu \mathrm{m}^{2}$ allows a flow change of $\Delta Q=3.4 \mathrm{l} / \mathrm{min}$ at $100 \mathrm{kPa}$ on an active chip area of only $2.3 \times 3.7 \mathrm{~mm}^{2}$.

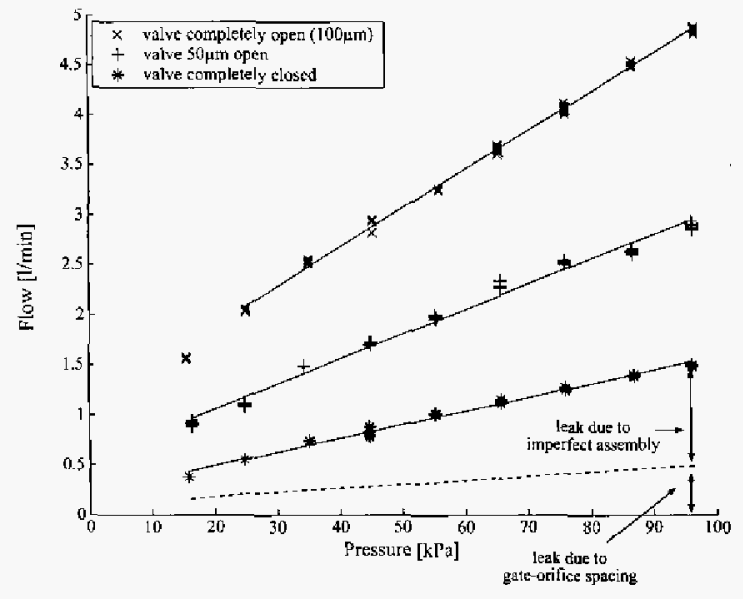

Figure 8: Measured pressure-flow characteristics. The solid lines are least-squares approximations of the flow in the supersonic regime. The dashed line indicates the expected leak caused by the spacing between orifice and gate.

The leak flow in the closed state can be divided into two parts. Approximately $30 \%$ is due to the gate-orifice spacing. The other $70 \%$ originate from the manual assembly and are caused by a $\sim 15 \mu \mathrm{m}$ leak between the glass substrate and the chip. The leak flow is considered acceptable for the intended application in IP-converters. The flow performance per footprint area is at least a factor ten higher than that of non-gate microvalves designed for similar flows [4].

\section{CONCLUSION \& OUTLOOK}

We presented the first fully micromachined out-of-plane knife gate microvalve, together with the characterization of the simplified thermal bimorph actuator and flow-pressure measurements. The flow performance per footprint area is increased by out-ofplane actuators and an out-of-plane orifice and is at least a factor ten higher than that of non-gate microvalves designed for similar flows. An active chip area of only $2.3 \times 3.7 \mathrm{~mm}^{2}$ allows a flow change of $\Delta Q=3.4 \mathrm{l} / \mathrm{min}$ at $100 \mathrm{kPa}$. Three different layouts of actuator-gate designs are introduced and discussed. The lack of thermal insulation prevented the thermal bimorph actuator from closing the valve completely by resistive heating. The valve flow was controlled gradually through the gate position using an extemal manipulator.

Future work will focus on improved actuation and device packaging.

\section{Acknowledgements}

This work has been carried out with the financial support of Vinnova through the Summit framework.

\section{References}

[1] P. J. Hesketh, J. S. Bintoro, and L. Luharuka, "Microvalve for fuel cells and miniature gas chromatographic system" in Sensors Update 13, J. K. H. Baltes, G. K. Fedder, Ed. Weinheim: WILEY-VCH Verlag GmbH \& Co. KGaA, 2004 , pp. 233-302.

[2] M. Huff, J. Gilbert, and M. Schmidt, "Flow Characteristics of a pressure balanced microvalve" in Proc. $7^{\text {th }}$ International Conference on Solid State Actuators (Transducers), Yokohama, Japan, 1993, pp. 98-101.

[3] W. van der Wijngaart, H. Ask, P. Enoksson, and G. Stemme, "A high-stroke, high-pressure electrostatic actuator for valve applications", Sensors and Actuators A (Physical), vol. A100, no. 2-3, p. 264, 2002.

[4] W. van der Wijngaart, A. Thorsén, and G. Stemme, "A seat microvalve nozzle for optimal gas flow capacity at large controlled pressure" in Journal of Micro Electro Mechanical Systems, 2005 .

[5] R. Zengerle, J. Wirtl, and H. Frisch, Patent DE 19546181 A1, C2, 1997.

[6] M. Walters, V. Dhuler, R. Mahadevan, A. Cowen, R. Wood, E. Hill, and I. Kao, "A silicon micromachined gate valve" in 1998 Solid-State Sensors and Actuators Symposium, South Carolina, USA, 1998.

[7] W. van der Wijngaart, A. S. Ridgeway, and G. Stemme, "A micromachined knife gate valve for high-flow pressure regulation applications" in Digest of Technical Papers, Transducers '03. 12th International Conference on Solid-State Sensors, Actuators and Microsystems, vol. 2. Boston, MA, USA: IEEE, 2003, pp. 1931-4. 